TITLE:

\title{
Photonic bands in two-dimensional microplasma arrays. I. Theoretical derivation of band structures of electromagnetic waves
}

\section{AUTHOR(S):}

Sakai, O; Sakaguchi, T; Tachibana, K

\section{CITATION:}

Sakai, O ...[et al]. Photonic bands in two-dimensional microplasma arrays. I. Theoretical derivation of band structures of electromagnetic waves. JOURNAL OF APPLIED PHYSICS 2007, 101(7): 073304.

ISSUE DATE:

2007-04-01

URL:

http://hdl.handle.net/2433/50123

\section{RIGHT:}

Copyright 2007 American Institute of Physics. This article may be downloaded for personal use only. Any other use requires prior permission of the author and the American Institute of Physics. 


\title{
Photonic bands in two-dimensional microplasma arrays. I. Theoretical derivation of band structures of electromagnetic waves
}

\author{
Osamu Sakai, ${ }^{\text {a) }}$ Takui Sakaguchi, and Kunihide Tachibana \\ Department of Electronic Science and Engineering, Kyoto University, Kyoto-daigaku Katsura, \\ Nishikyo-ku, Kyoto 615-8510, Japan
}

(Received 15 September 2006; accepted 22 January 2007; published online 6 April 2007)

\begin{abstract}
Two theoretical approaches appropriate for two-dimensional plasma photonic crystals reveal dispersions of propagating waves including photonic (electromagnetic) band gaps and multiflatbands. A modified plane-wave expansion method yields dispersions of collisional periodical plasmas, and the complex-value solution of a wave equation by a finite difference method enables us to obtain dispersions with structure effects in an individual microplasma. Periodical plasma arrays form band gaps as well as normal photonic crystals, and multiflatbands are present below the electron plasma frequency in the transverse electric field mode. Electron elastic collisions lower the top frequency of the multiflatbands but have little effect on band gap properties. The spatial gradient of the local dielectric constant resulting from an electron density profile widens the frequency region of the multiflatbands, as demonstrated by the change of surface wave distributions. Propagation properties described in dispersions including band gaps and flatbands agree with experimental observations of microplasma arrays. (c) 2007 American Institute of Physics.
\end{abstract}

[DOI: $10.1063 / 1.2713939]$

\section{INTRODUCTION}

Dispersion relations of propagating electromagnetic waves in nonmagnetized plasma can be modified if bulk plasma is replaced by a microplasma array, which is analogically understood from the extensive studies of photonic crystals. ${ }^{1,2}$ As shown in the theoretical work of onedimensional periodical plasma layers by Faith et al. ${ }^{3}$ and reconfirmed by Kalluri ${ }^{4}$ and Hojo and Mase, ${ }^{5}$ forbidden bands for wave propagation are formed beyond the bulk cutoff frequency (electron plasma frequency) due to periodicity, where one can refer to such a functional structure as a "plasma photonic crystal." More drastic modification of dispersion relations is possible when periodicity becomes two dimensional, and one example was found in a numerical study of beaming phenomena. ${ }^{6}$

Our previous reports ${ }^{7,8}$ demonstrated experimental verifications of the unique features of plasma photonic bands in a two-dimensional periodical microplasma array in tens of gigahertz frequencies, such as band gap ${ }^{7}$ and abnormal refraction ${ }^{8}$ that had not been reported even theoretically. In technical applications, the dynamic properties of plasmas including the rapid change of dielectric constant and periodical length by tuning individual plasma ignition are quite beneficial for a high-functional electromagnetic wave controller. These experimental results were explained by theoretical analysis using a normal plane-wave expansion method ${ }^{7}$ or a direct solution of a wave equation. ${ }^{8}$

In this report, further expansion of these two theoretical methods uncovers the overview of the entire dispersion relation and several specific features of wave propagation in a two-dimensional periodical plasma array. Such a theory is associated with metallic photonic crystals that have been investigated in several ways. ${ }^{9-13}$ In both plasma and metallic

${ }^{a)}$ Electronic mail: osakai@kuee.kyoto-u.ac.jp photonic crystals, the dielectric constant is given by the Drude model with plasma frequency, and so a similar calculation technique used in a metallic photonic crystal system in the photon range can be adapted to our case to a certain extent. However, electron elastic collision against neutral particles is comparable to the plasma frequency in a microplasma working around ambient atmospheric pressure, whereas the inverse of the electron relaxation time is much smaller than the plasma frequency in metals. ${ }^{14}$ For instance, microplasma in a plasma display panel has an electron density ranging from $10^{13}$ to $10^{14} \mathrm{~cm}^{-3}$, ${ }^{15}$ which corresponds to the electron plasma frequency of $\omega_{\mathrm{pe}} / 2 \pi=28-90 \mathrm{GHz}$. However, electron elastic collision frequency $\nu_{\mathrm{m}} / 2 \pi$ is in a similar range to $\omega_{\mathrm{pe}} / 2 \pi$ in a $\mathrm{Ne} / \mathrm{Xe}$ mixture of 400-600 Torr. A more distinctive difference from the metallic cases is the electron density profile existing inside a plasma; the local dielectric constant changes significantly inside one plasma, which makes an approximation method applicable to metallic cases inefficient.

In this report we demonstrate two theoretical approaches to plasma photonic crystals to fulfill these requirements: modification of the plane-wave expansion method and direct solution of the wave equation with complex-value electric fields. Rigorous treatment of the Drude model with significant electron elastic collision frequency is applied to the plane-wave expansion method for the infinite size of an array, and the derived band diagrams demonstrated several features both similar to and different from the usual photonic bands created by a dielectric periodical structure with constant dielectric constant over frequencies. Here we investigate several influences of high electron elastic collision frequency. Another approach that uncovers the wave propagation properties is the direct solution of the wave equation with complex-value electric fields by the finite difference method that yields dispersion relations (band dia- 
grams) numerically, even if the unit structure in periodicity is too complicated to be solved by the plane-wave expansion method. In this article we examine the effects of electron density gradient or the local change of plasma frequency inside a plasma on band diagrams. These theoretical calculations are compared with experimental results that include observations of band gap and flatband properties.

In Sec. II, the extended theory of the plane-wave expansion method and the numerical solution technique of wave equation using complex-value wave fields are described. In Sec. III, these two theoretical methods yield specific examples of band diagrams attributed to the periodicity of plasmas. In Sec. IV, these theoretical results are compared with experimental observations, followed by a summary in Sec. V.

\section{THEORETICAL APPROACHES TO DERIVE DISPERSION RELATIONS}

\section{A. Plane-wave expansion method with Drude model in a collisional plasma}

The plane-wave expansion method has been widely used to analytically derive photonic band diagrams of two- and three-dimensional dielectric periodical structures. ${ }^{16,17}$ A spatially periodical dielectric coefficient, which is constant over a certain frequency range, is divided into the summation of spatial Fourier coefficients, and an assumption that multiplane-waves are superposed leads to dispersion relations by solving an eigenvalue problem.

When a solid dielectric array is replaced by multimicroplasmas, the dielectric constant depends on wave frequency $\omega / 2 \pi$, such as in the Drude type, and the normal plane-wave expansion method is ineffective. Kuzmiak and Maradudin developed a plane-wave expansion method applicable to the photonic band structures of metallic components, ${ }^{9}$ and here we use a similar technique to solve the eigenvalue problem and further investigate cases similar to our experimental conditions.

When the field components in electromagnetic waves are proportional to $\exp [j(\omega t-k \cdot x)]$, where $\boldsymbol{k}$ and $\boldsymbol{x}$ are the complex wave number and spatial position vector, respectively, the dielectric constant as a function of frequency $\varepsilon_{\mathrm{p}}(\omega)$ inside a cold plasma column with electron plasma frequency $\left(\omega_{\text {pe }}\right)$ is written as

$$
\varepsilon_{\mathrm{p}}=1-\left(\frac{\omega_{\mathrm{pe}}}{\omega}\right)^{2} \frac{1}{1-j\left(\nu_{\mathrm{m}} / \omega\right)},
$$

where $\nu_{\mathrm{m}}$ is the electron elastic collision frequency determined by neutral gas pressure and elastic collision cross section. In metal cases, ${ }^{9}$ a similar value $(\gamma)$ to $\nu_{\mathrm{m}}$ was used as an inverse of electron relaxation time, and $\gamma$ was much smaller than $\omega$ and $\omega_{\mathrm{pe}}$ in the calculation of Ref. 9. On the other hand, in our cases, $\nu_{\mathrm{m}}$ is comparable to $\omega$ and $\omega_{\mathrm{pe}}$, where electron density $n_{\mathrm{e}}$ is around $10^{13} \mathrm{~cm}^{-3}$ at a gas pressure around atmospheric pressure, and such a parameter region is explored in the following. We set the dielectric region with dielectric constant $\varepsilon_{\mathrm{d}}$ outside the plasma column, since in experiments we frequently confine discharge gases and plasmas in a dielectric container. Using Eq. (1), the position vector at the site of lattice point $\boldsymbol{x}_{\|}$, and translation vector $\boldsymbol{G}_{\|}$ $\left(=h_{1} \boldsymbol{b}_{1}+h_{2} \boldsymbol{b}_{2}\right.$, where $\boldsymbol{b}_{1}$ and $\boldsymbol{b}_{2}$ are the primitive translation vectors of the reciprocal lattice and $h_{1}$ and $h_{2}$ are arbitrary integers), spatially and frequency-dependent dielectric constant $\varepsilon\left(\boldsymbol{x}_{\|} \mid \omega\right)$ in a square lattice is expressed by Fourier coefficients $\hat{\varepsilon}\left(\boldsymbol{G}_{\|}\right)$as

$$
\varepsilon\left(\boldsymbol{x}_{\|} \mid \omega\right)=\sum_{G_{\|}} \hat{\varepsilon}\left(\boldsymbol{G}_{\|}\right) e^{j \boldsymbol{G}_{\|} \cdot \boldsymbol{x}_{\|}}
$$

and

$$
\begin{aligned}
\hat{\varepsilon}\left(\boldsymbol{G}_{\|}\right) & =\varepsilon_{\mathrm{d}}-f\left[1-\varepsilon_{\mathrm{d}}-\frac{\omega_{\mathrm{pe}}{ }^{2}}{\omega\left(\omega-j \nu_{\mathrm{m}}\right)}\right], \quad \boldsymbol{G}_{\|}=0 \\
& =f\left[1-\varepsilon_{\mathrm{d}}-\frac{\omega_{\mathrm{pe}}^{2}}{\omega\left(\omega-j \nu_{\mathrm{m}}\right)}\right] \frac{2 J_{1}\left(\boldsymbol{G}_{\|} R\right)}{\left(\boldsymbol{G}_{\|} R\right)}, \quad \boldsymbol{G}_{\|} \neq 0,
\end{aligned}
$$

where $f=\pi R^{2} / a^{2}$ is the filling fraction and $R$ is the radius of the cross section of a plasma column with lattice constant $a$. This frequency-dependent complex value of $\varepsilon\left(\boldsymbol{x}_{\|} \mid \omega\right)$ complicates the eigenvalue problem. Linearization of this generalized eigenvalue problem of the complex matrix, ${ }^{9}$ whose details will be shown in the Appendix, yields dispersion relations by using 25 plane waves. This number of waves is limited by our computational hardware. Possible deficiency of this number might lead to problems of convergence of the calculated dispersions, as pointed out by Kuzmiak and Maradudin, ${ }^{9}$ which is one reason why we develop another method to derive dispersion relations in Sec. II B. Note that $\omega$ is a complex number, and its real part is derived to display a band diagram, and the imaginary part represents the damping rate of the corresponding mode.

\section{B. Direct solution of wave equation using complex electric fields}

Direct numerical analysis of Maxwell equations is usually by a finite difference time-domain (FDTD) method, ${ }^{10,18}$ which successfully gave rise to the band diagrams of a twodimensional metallic photonic crystal. What we use here to obtain band diagrams numerically is a different method involving the complex values of electromagnetic fields that enables us to obtain a static solution of the fields in shorter central processing unit (CPU) time. A description of the method is found in Refs. 8 and 19, and here we mention its details.

From the Maxwell equations, the elimination of magnetic fields leads to a wave equation composed of electric fields $\boldsymbol{E}$, external current density $\boldsymbol{J}_{\text {ext }}$, permeability $\mu$, and complex dielectric constant $\varepsilon$ with permittivity of free space $\varepsilon_{0}$, written as

$$
\nabla \times \nabla \times \boldsymbol{E}-\omega^{2} \mu \varepsilon \varepsilon_{0} \boldsymbol{E}=j \omega \mu \boldsymbol{J}_{\mathrm{ext}},
$$

which is spatially discretized (in our case, $20 \times 20$ square meshes in one lattice) based on the finite difference method. We note that $\varepsilon$ is expressed as $\varepsilon_{\mathrm{p}}(\omega)$ in Eq. (1) inside a plasma. Two-dimensional complex-value electric field in the form of $\exp [j(\omega t-k \cdot x)]$ is considered, and so the TE mode in which an electric field is parallel to a two-dimensional lattice plane can be analyzed when electric field vectors are 
vertical to plasma columns, in applications of the Bloch theorem to electric and magnetic (or gradient of electric) fields on the boundaries of a lattice in the form of

$$
\begin{aligned}
& \boldsymbol{E}\left(x_{0}+a\right)=\boldsymbol{E}\left(x_{0}\right) \exp \left(-j k_{x} a\right), \\
& \boldsymbol{E}\left(y_{0}+a\right)=\boldsymbol{E}\left(y_{0}\right) \exp \left(-j k_{y} a\right)
\end{aligned}
$$

and

$$
\begin{aligned}
& \frac{d \boldsymbol{E}}{d \boldsymbol{x}}\left(x_{0}+a\right)=\frac{d \boldsymbol{E}}{d \boldsymbol{x}}\left(x_{0}\right) \exp \left(-j k_{x} a\right), \\
& \frac{d \boldsymbol{E}}{d \boldsymbol{x}}\left(y_{0}+a\right)=\frac{d \boldsymbol{E}}{d \boldsymbol{x}}\left(y_{0}\right) \exp \left(-j k_{y} a\right),
\end{aligned}
$$

where $\left(x_{0}, y_{0}\right)$ is a position vector on the boundary of lattices. In the case of the TM mode analysis, two-dimensional magnetic fields will be solved numerically.

From these boundary conditions, the two-dimensional wave number of a propagating wave $\left(k_{x}, k_{y}\right)$ is specified. The external current source is hypothetically set within a lattice. Then Eq. (4) is solved using the finite difference method at each discrete frequency from 0 to $150 \mathrm{GHz}$, and local wave power density is chosen as a cost function to detect a dispersion branch. That is, when one set of frequency and wave number is matched with a propagating wave condition, the cost function represents resonancelike peaking with a very narrow frequency region (usually less than $0.1 \mathrm{GHz}$ width) and with a nonlocal electric field pattern independent of the position of the hypothetical current source. On the other hand, when another set is not along the dispersion branch, the electric field profile only shows a near-field pattern localized around the hypothetical current source with a very small cost function value. A complex value of the electric field includes phase information, and so the wave number assumed in Eqs. (5) and (6) can be reconfirmed by a spatially differential value of the phase $(\boldsymbol{k} \cdot \boldsymbol{x})$ at the resonancelike frequency. In this method, no derivation of a spatial Fourier coefficient is required, and so an arbitrary structure inside a lattice such as a complicated plasma shape and an arbitrary $\varepsilon$ profile can be treated.

This method does not use time-domain discretization, unlike the FDTD method. In the case of the FDTD method, time evolution of propagating waves in media is converted into frequency spectra. To deal with the wide frequency range simultaneously, it is required to perform auxiliary calculation to reinforce the dispersive dependence of the dielectric constant such as shown in Eq. (1), and such a scheme is referred to as frequency-dependent FDTD method. ${ }^{20}$ In our method used here, a monochromatic wave at one frequency is assumed in each calculation step with a corresponding and precise value of the dielectric constant from Eq. (1). In other words, the frequency step which we set for searching wave propagation is crucial to assure the entire calculation accuracy. A narrower frequency step will yield a more accurate determination of propagating wave, although more CPU time is required.

Using this scheme, we calculated band diagrams with $\nu_{\mathrm{m}} \ll \omega$, that is, a collisionless case. When $\nu_{\mathrm{m}}$ is introduced as

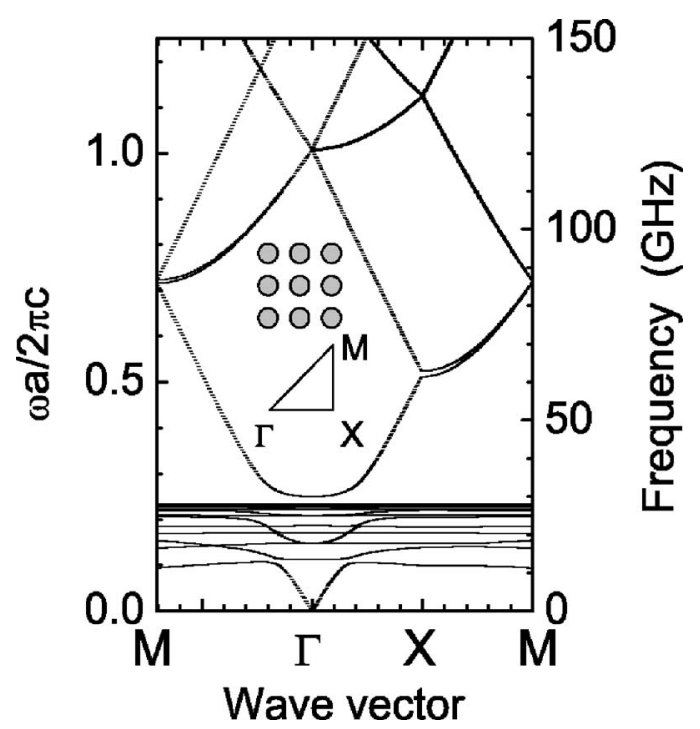

FIG. 1. Band diagram of TE mode by plane-wave expansion method in the case of lattice constant $a$ of $2.5 \mathrm{~mm}$ with 1.0 -mm-diameter collisionless plasma column of $n_{\mathrm{e}}=10^{13} \mathrm{~cm}^{-3}$.

a finite value comparable to $\omega$ and $\omega_{\text {pe }}$, note that the resonancelike frequency is searched for as a complex value for a real wave number, like in the cases of the plane-wave expansion method described in Sec. II A. If we consider spatial wave damping of the static propagation in a finite region, a complex wave number is derived for a real value of frequency. This method enables us to take such a flexible approach.

\section{DISPERSIONS CALCULATED ANALYTICALLY AND NUMERICALLY}

\section{A. Overview of plasma photonic bands and collisional effects}

Figure 1 shows a band diagram of the TE mode by the plane-wave expansion method in the case of lattice constant $a$ of $2.5 \mathrm{~mm}$ with a $1.0-\mathrm{mm}$-diameter plasma column of $n_{\mathrm{e}}$ $=10^{13} \mathrm{~cm}^{-3}$. Here the collisionless case with $\varepsilon_{\mathrm{d}}=1$ is investigated first for simplicity. There are two significant features which characterize a band diagram of a plasma photonic crystal. First, band gaps are formed whose width increases where their frequencies are close to $\omega_{\text {pe }}$ due to the dispersion shown in Eq. (1). For instance, the first band gap in the $\Gamma-X$ direction around $62 \mathrm{GHz}$, where $\varepsilon_{\mathrm{p}}(\omega) \sim 0.80$, is clearly observed, and the first band gap in the $\Gamma-M$ direction around $87 \mathrm{GHz}$ with $\varepsilon_{\mathrm{p}}(\omega) \sim 0.90$ is also observable. However, the second gap in the $\Gamma-X$ direction around $134 \mathrm{GHz}$ is almost closed since the frequency is quite far from the plasma frequency $\left(\omega_{\mathrm{pe}} / 2 \pi=28 \mathrm{GHz}\right)$, and $\varepsilon_{\mathrm{p}}(\omega)(=0.96)$ is close to the vacuum value. In conventional photonic crystals in the combination of dielectric components and vacuum, a ratio of $\varepsilon$ can be set to be quite large (e.g., >5), so that clearer band gaps emerge. On the other hand, the $\varepsilon$ ratio in a plasma photonic crystal is only large near the plasma frequency; the $\varepsilon$ ratio is 2 at $40 \mathrm{GHz}$ for this plasma frequency. That is, significant features distinguished from vacuum propagation are expected around the plasma frequency. In other words, periodical plasmas combined with dielectrics, where the $\varepsilon$ 
ratio is large in the entire frequency range and it is enhanced near the plasma frequency, may open other parameter ranges of band properties.

The second and a unique feature of the plasma photonic crystal exhibited in Fig. 1 is that multiflatbands emerge at $\omega<\omega_{\mathrm{pe}}$. In this frequency region, $\varepsilon(\omega)$ is negative inside a plasma column. Along these flatbands, surface plasma waves, which are surface plasmon polaritons in the notation of metallic photonic crystals, are present with very localized fields around a plasma column. In the TE mode where electric fields are perpendicular to plasma columns, electric field component normal to the plasma surface is possible along a circular plasma column, and if near fields excited around the periodical plasma columns can propagate through them in phase, wave propagation is possible on a flatband. This feature is quite different from the case of a large-volume plasma, where a longitudinal electric field only exists at $\omega$ $=\omega_{\mathrm{pe}}$ with zero wave number on a bulk plasma surface. Each flatband has a different azimuthal mode number and an electromagnetic field profile along a plasma column, as pointed out by Ito and Sakoda. ${ }^{18}$ Related features of field distribution will be demonstrated later in the numerical results of the finite difference method.

When $\nu_{\mathrm{m}}$ has a finite value comparable to $\omega=\omega_{\mathrm{pe}}$, several changes in the dispersion relation are induced as well as an increase of wave damping through electron elastic collisions. Figure 2(a) shows a band diagram of the TE mode by the plane-wave expansion method in a similar case to Fig. 1, except that $\nu_{\mathrm{m}}=0.5 \omega_{\mathrm{pe}}$. The top frequency of the flatbands decreases because the equivalent cutoff frequency of bulk plasmas is modified due to $\nu_{\mathrm{m}}$; from Eq. (1), the real part of $\varepsilon_{\mathrm{p}}(\omega)$ is expressed as

$$
\operatorname{Re}\left(\varepsilon_{\mathrm{p}}\right)=1-\frac{\omega_{\mathrm{pe}}^{2}}{\omega^{2}+\nu_{\mathrm{m}}^{2}} .
$$

As far as band gaps are concerned, $\nu_{\mathrm{m}}$ hardly affects them due to the Drude model function expressed by Eq. (1). On the whole, the propagation band property indicates similarity to the collisionless one with some small changes. $\nu_{\mathrm{m}}$ rather affects wave damping, as studied by Kuzmiak and Maradudin ${ }^{9}$ and discussed in our previous work associated with integrated microplasmas. ${ }^{8}$

Figure 2(b) shows a band diagram of the TM mode in a similar case to Fig. 2(a). In TM modes, electric fields are only present along the plasma column axis without normal components to the surface and screened out at $\operatorname{Re}\left(\varepsilon_{\mathrm{p}}\right)<0$. In other words, surface plasma waves, which have a longitudinal component, are only excited where $\operatorname{Re}\left(\varepsilon_{\mathrm{p}}\right) \sim 0$ in Eq. (7) and cannot match the electric fields of this polarity at $\operatorname{Re}\left(\varepsilon_{\mathrm{p}}\right)<0$, where multiflatbands exist in the case of the TE mode.

When we increase electron elastic collisions by raising the gas pressure, the flatband structure vanishes in the range where $\nu_{\mathrm{m}}>2 \omega_{\mathrm{pe}}$. Figure 3 displays a band diagram in the case where $\nu_{\mathrm{m}}=2 \omega_{\mathrm{pe}}$. There is no detected flatband for two reasons: high collisionality prevents surface plasma waves from azimuthally forming standing waves around a plasma column and high collisionality enables waves to propagate into the core of the plasma where no azimuthal structure with

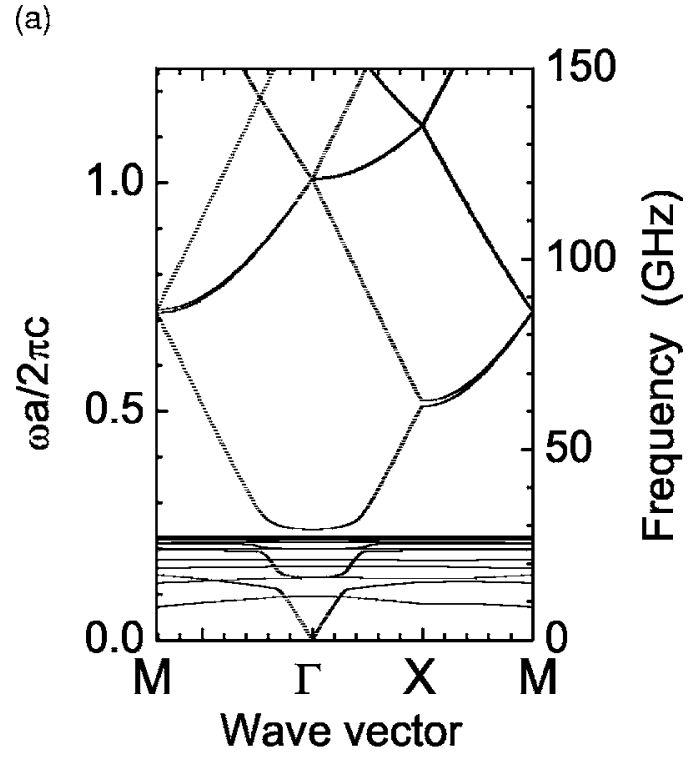

(b)

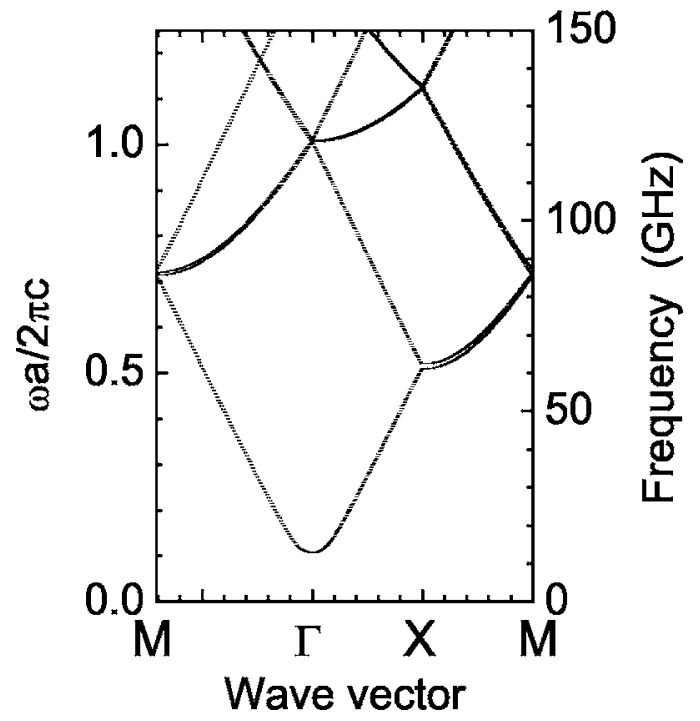

FIG. 2. (a) Band diagram of TE mode by plane-wave expansion method in similar case to Fig. 1 except that $\nu_{\mathrm{m}}=0.5 \omega_{\mathrm{pe}}$. (b) Band diagram of TM mode in similar case to (a).

a symmetric axis is possible. Another feature observed in Fig. 3 is that band gaps in each direction become narrower since the equivalent dielectric constant expressed in Eq. (7) gets close to the vacuum condition; for instance, in the vicinity of the first band gap in the $\Gamma-X$ direction around $62 \mathrm{GHz}, \operatorname{Re}\left[\varepsilon_{\mathrm{p}}(\omega)\right] \sim 0.89$. Note that wave damping through collisional plasmas is not so heavy when $\omega$ is much larger than $\omega_{\text {pe }}$, which was shown in our previous report. ${ }^{8}$ Wave cutoff properties at unidirectional band gaps are observable experimentally, although the band gap width becomes smaller by electron elastic collisions enhanced in high gas pressure plasmas.

The condition of $\omega_{\mathrm{pe}}$ studied in this case is $\omega_{\mathrm{pe}} a / 2 \pi c$ $\sim 0.25$, which is within our experimental parameters. ${ }^{7,8}$ If $\omega_{\text {pe }}$ increases due to $n_{\mathrm{e}}$ enhancement, the flatband region expands toward the upper frequency, and similar band structures to those calculated in Ref. 9 will be observable. Since the $\nu_{\mathrm{m}} / \omega_{\mathrm{pe}}$ ratio is high in plasmas in comparison with me- 


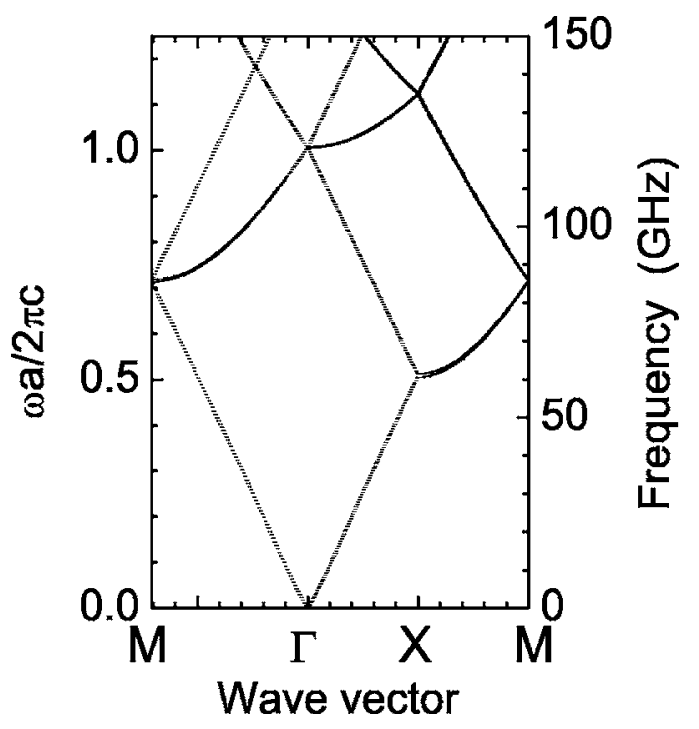

FIG. 3. Band diagram of TE mode by plane-wave expansion method in similar case to Fig. 1 except that $\nu_{\mathrm{m}}=2.0 \omega_{\mathrm{pe}}$.

tallic cases, heavy wave damping due to the imaginary part of the wave number below $\omega_{\text {pe }}$ might be an obstacle in applications to wave controllers. If $\nu_{\mathrm{m}}$ can be fixed to a lower value by such optimized parameters as gas species and plasma production schemes, higher $\omega_{\mathrm{pe}}$ will lead to various (and complicated) band structures.

\section{B. Effects of electron density gradient on plasma photonic bands}

Another important feature of a plasma photonic crystal, the spatially continuous change of $\varepsilon(\omega)$, is investigated by the direct solution of the wave equation using the finite difference method. Figure 4(a) shows a band diagram of the TE mode by a direct solution of Eq. (4) using complex-value fields and the finite difference method for lattice constant $a$ of $2.5 \mathrm{~mm}$ with a 1.0 -mm-diameter collisionless plasma column of $n_{\mathrm{e}}=10^{13} \mathrm{~cm}^{-3}$; the condition is similar to Fig. 1 . There is very good consistency between Figs. 1 and 4(a). That is, both methods play a role in deriving band diagrams of plasma photonic crystals. This method in which Fourier coefficients are not required enables us to obtain band diagrams of more specific cases. Based on the Bessel function $J_{0}$, the calculated band diagram in the case of gradual $n_{\mathrm{e}}$ profiles is shown in Fig. 4(b). Here, $n_{\mathrm{e}}(0)$ in the center is $10^{13} \mathrm{~cm}^{-3}$ and $n_{\mathrm{e}}(r)=n_{\mathrm{e}}(0) J_{0}\left(r / r_{\text {edge }}\right)$, where $r$ is the radial position from the center and $r_{\text {edge }}$ is set so that the full width at half maximum is $1.0 \mathrm{~mm}$, as schematically shown in Fig. 4(b). A radial $n_{\mathrm{e}}$ profile with $J_{0}$ function is usually observed in a positive column of a glow discharge. This result is almost similar to Fig. 4(a) where the $n_{\mathrm{e}}$ profile is in slab form, except for the broadening of the flatband region toward lower frequencies. This broadening of the frequency range of flatbands is attributed to the density gradient, since each level of $n_{\mathrm{e}}$ corresponds to different cutoff conditions ( $\omega$ $\left.=\omega_{\mathrm{pe}}\right)$ in an individual column. That is, for instance, a flatband around $9 \mathrm{GHz}$ results from the low $n_{\mathrm{e}}\left(\sim 10^{12} \mathrm{~cm}^{-3}\right)$ region in the edge and that around $25 \mathrm{GHz}$ results from the high $n_{\mathrm{e}}\left(\sim 10^{13} \mathrm{~cm}^{-3}\right)$ region in the core. In higher frequen- (a)
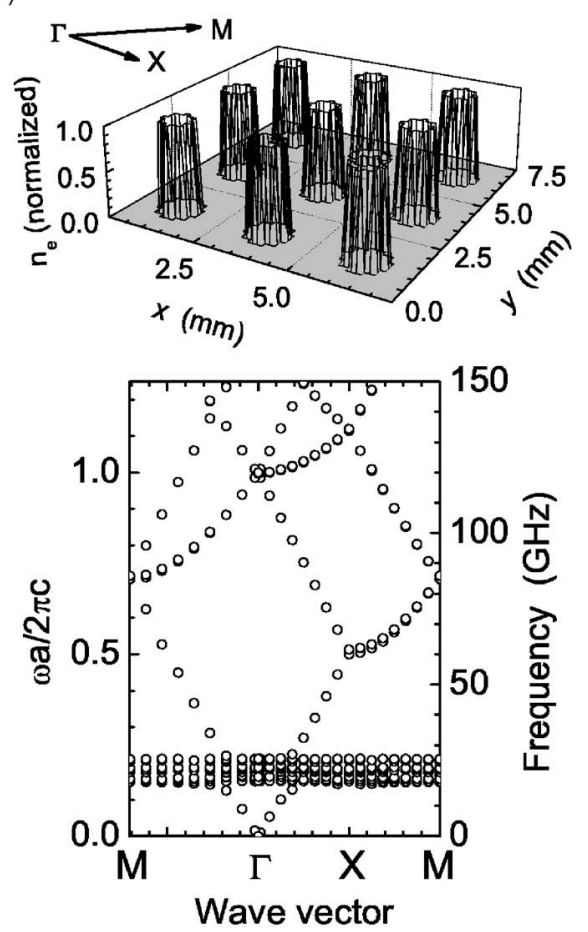

(b)
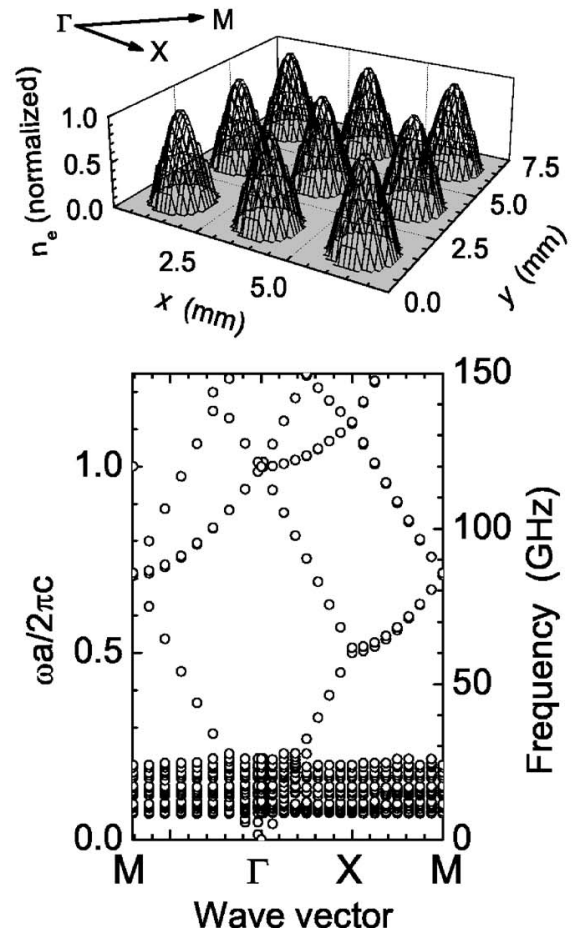

FIG. 4. (a) Band diagram of TE mode by direct solution method of wave equation using complex fields in the case of lattice constant $a$ of $2.5 \mathrm{~mm}$ with 1.0-mm-diameter collisionless plasma column of $n_{\mathrm{e}}=10^{13} \mathrm{~cm}^{-3} \cdot n_{\mathrm{e}}$ profile is slab shaped. (b) Band diagram of TE mode by direct solution method in the case of lattice constant $a$ of $2.5 \mathrm{~mm}$ with collisionless plasma column in which $n_{\mathrm{e}}$ profile is in the shape of $J_{0}$, where the maximum $n_{\mathrm{e}}$ $=10^{13} \mathrm{~cm}^{-3}$ and full width at half maximum is $1.0 \mathrm{~mm}$.

cies dispersion properties such as band gaps are not so affected by changes of the $n_{\mathrm{e}}$ profile, partly because the integrated refractive index in a unit lattice is more effective for such features. 

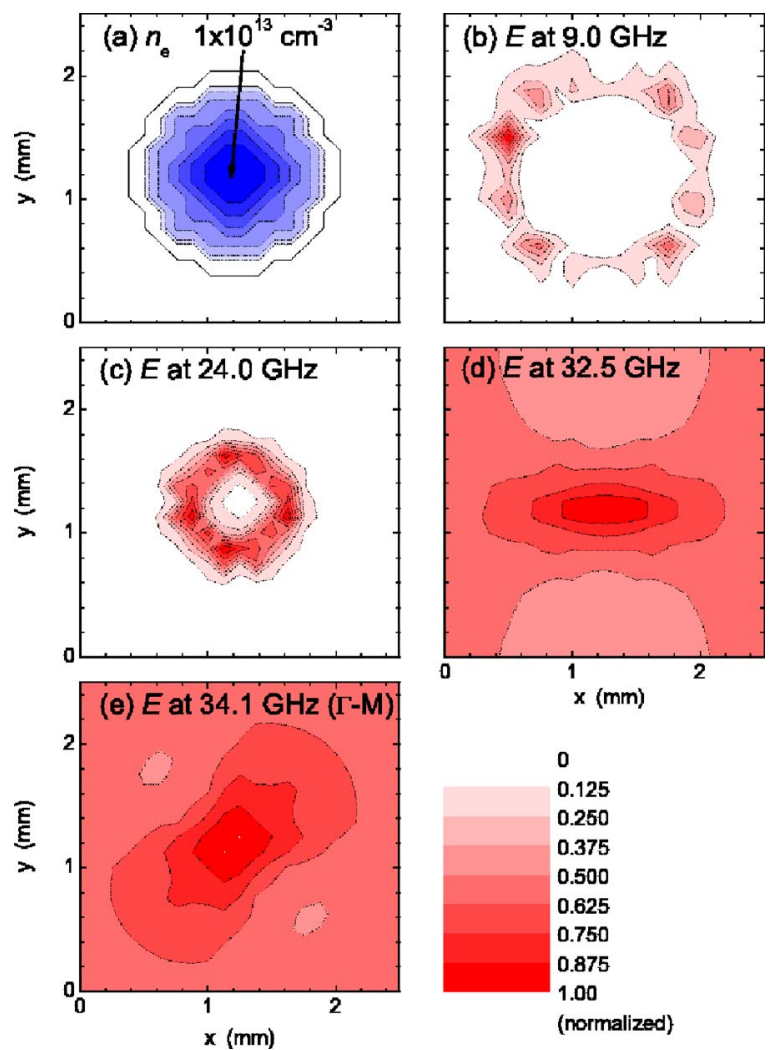

FIG. 5. (Color online) Parameter profiles in one lattice in similar condition in Fig. 4(b). (a) Assumed $n_{\mathrm{e}}$ profile in one lattice, based on the Bessel function $J_{0}$. Calculated profiles of electric fields normalized in amplitude in the case of $k_{x} a / 2 \pi=0.25$ and $k_{y}=0$ (b) at $9.0 \mathrm{GHz}$, (c) at $24.0 \mathrm{GHz}$, (d) at $32.5 \mathrm{GHz}$, and (e) in the case of $k_{x} a / 2 \pi=0.17$ and $k_{y} a / 2 \pi=0.17$ at $34.1 \mathrm{GHz}$.

Propagating electromagnetic fields in a plasma photonic crystal vary drastically and depend on a band branch. Figure 5 exhibits electric field profiles derived as a subproduct in the calculation of Fig. 4(b) at $k_{x} a / 2 \pi=0.25$ and $k_{y}=0$, except for Fig. 5(e) at $k_{x} a / 2 \pi=0.17$ and $k_{y} a / 2 \pi=0.17$. Note that these $E$ values are absolutes including both real and imaginary components. These specific points in the $\left(k_{x}, k_{y}\right)$ plane have no special meaning; these are usual wave number vectors along the first band in the $\Gamma-X$ or $\Gamma-M$ direction. The assumed $n_{\mathrm{e}}$ profile is drawn again in Fig. 5(a) as a contour plot whose radial dependence reflects a $J_{0}$ function. Figure 5(b) shows the $E$ profile at $9.0 \mathrm{GHz}$, which is distributed on the edge of the plasma column as a surface wave. When the frequency is elevated to $24.0 \mathrm{GHz}$, which is slightly less than $\omega_{\text {pe }} / 2 \pi$ at the central point, $E$ penetrates the inner region but not the core. The $E$ profile in this case also suggests surface wave propagation. From Figs. 5(b) and 5(c) it is clarified that a surface wave propagates just out of the layer at $\omega=\omega_{\mathrm{pe}}$. At $32.5 \mathrm{GHz}$, which is slightly above $\omega_{\mathrm{pe}} / 2 \pi$ and shown in Fig. $5(\mathrm{~d}), E$ exists selectively inside the plasma column due to lower positive $\varepsilon$ than in the surrounding vacuum region. Figure 5(e) shows a similar case to Fig. 5(d) with a different propagation direction $(\Gamma-M)$. These contours demonstrate that band diagrams calculated analytically and numerically are well understood from a reasonable physical point of view.
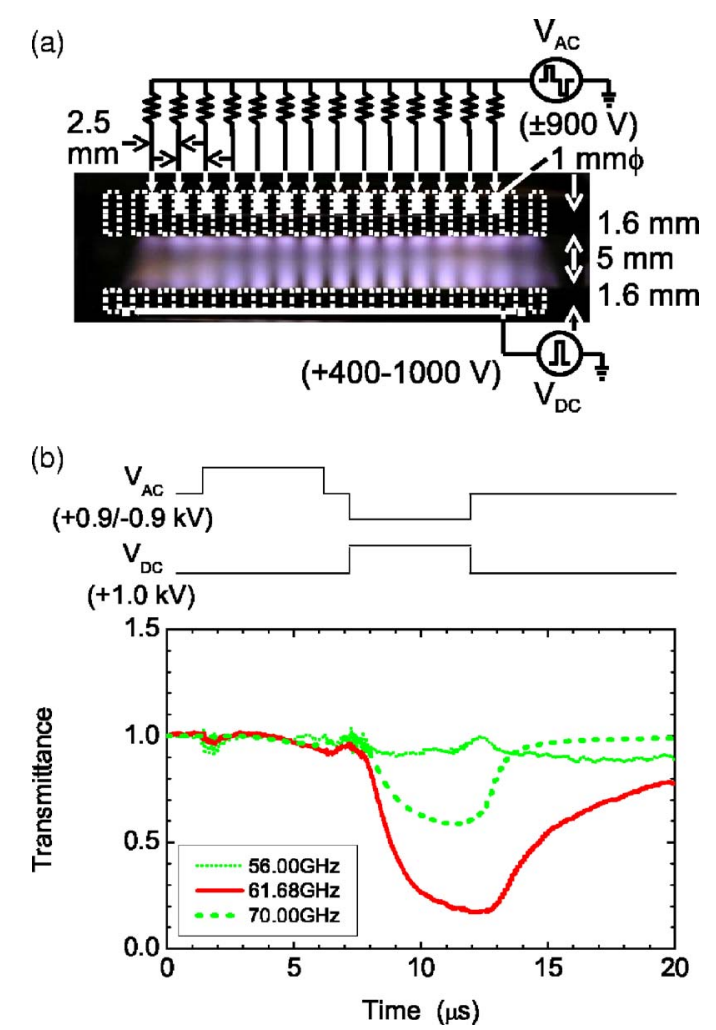

FIG. 6. (a) (Color online) Side view of the multicapillary electrode system of $17 \times 30$ array in $a=2.5 \mathrm{~mm}$ and $R=0.5 \mathrm{~mm}$ and visible emission from plasma photonic crystal. (b) Time evolutions of detected millimeter signal in TE mode transmitted through microplasma array region. Inset lines indicate voltage pulses imposed on electrodes.

\section{COMPARISON WITH EXPERIMENTAL OBSERVATION OF PROPAGATING WAVES}

\section{A. Photonic band gap formed above electron plasma frequency}

We assume that a two-dimensional circular microplasma array in a square lattice is produced in free space. Above the electron frequency, the dielectric constant in a plasma is positive and less than 1 , which leads to spatial periodicity of the dielectric constant. The first band gap in the $\Gamma-X$ direction is predicted to exist around $\omega a / 2 \pi c=0.5$ in Figs. $1-4$.

Experiments were performed to investigate dispersions in plasma photonic crystals by millimeter wave injection into a microplasma array region. They were generated using a multicapillary electrode system ${ }^{21}$ where $a=2.5 \mathrm{~mm}$ and $R$ $=0.5 \mathrm{~mm}$, as schematically shown in Fig. 6(a), with visible emission from the microplasma array. A bipolar voltage pulse was applied on an electrode array, and twodimensional uniformity was maintained using ballast resistance $(47 \mathrm{k} \Omega)$ in each electrode of the array. A synchronous monopolar voltage pulse was applied on an electrode plate on the other side. A $17 \times 30$ uniform array was successfully formed in the transverse He flow of $21 / \mathrm{min}$ at 200 Torr. A more detailed description of the electrode structure will be reported in the following paper. ${ }^{22}$ Figure 6(b) shows the detected millimeter signal in the TE mode transmitted from the other side in the $\Gamma-X$ direction through 30 microplasma rows. The setup for millimeter launching and detection was described in previous reports ${ }^{7,8}$ and in the following paper. ${ }^{22}$ 


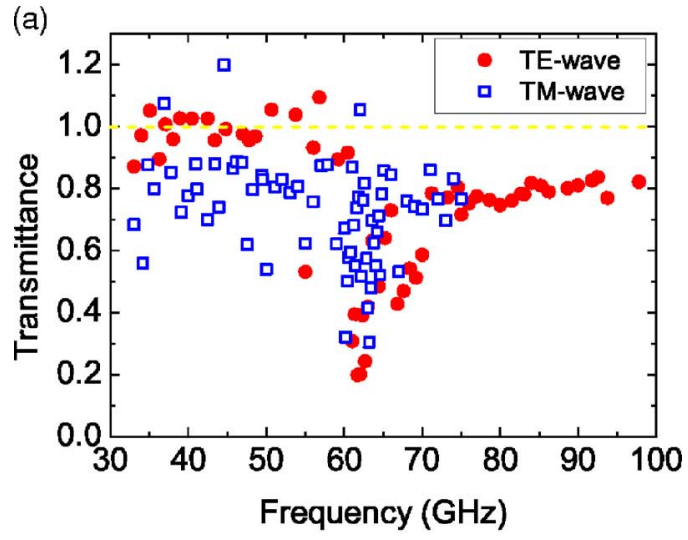

(b)

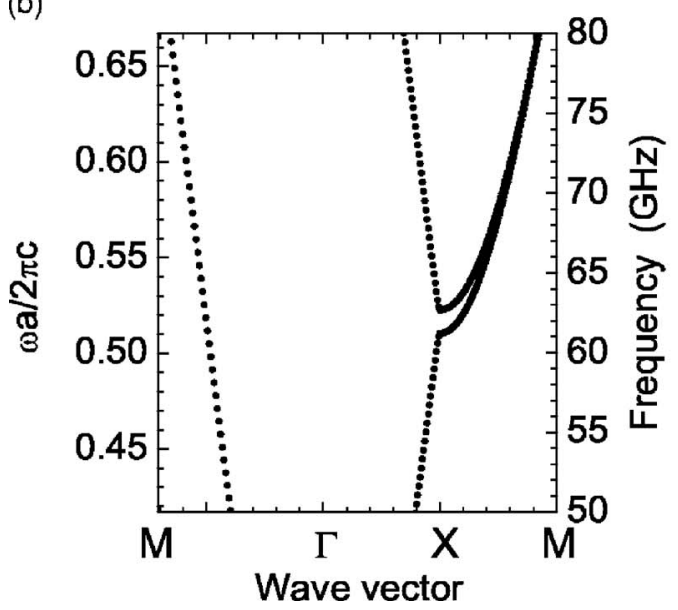

FIG. 7. (a) (Color online) Transmittance of TE and TM mode signals as a function of millimeter frequency. Experimental setup is described in Fig. 6. (b) Band diagram enlarged from Fig. 2(a) around the first band gap in the $\Gamma-X$ direction.

Note that metal apertures were set both at the entrance and the exit of the array region to prevent millimeter waves, which penetrate the area outside the microplasma array, from entering the detector. At $61.7 \mathrm{GHz}$, the signal was reduced by more than $80 \%$, whereas those at the other two frequencies showed a slight decrease during discharges.

Figure 7(a) displays the transmittance of the TE and TM mode signals in the $\Gamma-X$ direction as a function of frequency $\omega / 2 \pi$ and clearly showed a drop of transmittance around 60-63 GHz. Figure 7(b) illustrates the dispersion relation enlarged from Fig. 2(a) around the first band gap in the $\Gamma-X$ direction. The drop regions of both the TE and TM modes in Fig. 7(a) are consistent with the first band gap suggested in Fig. 7(b). Other attenuation in the second band beyond the band gap was also observed and attributed to a matching condition between the waves from the free space and the possible field patterns in the corresponding mode, as suggested in Ref. 23 and discussed in the following paper. ${ }^{22}$

\section{B. Abnormal refraction near electron plasma frequency}

Near and just above the electron plasma frequency, refractive index in the case of TE mode gets close to zero with phase velocity infinity and with group velocity zero. That is, the ratio of refractive index between plasma and vacuum regions is quite high, and several unusual events may happen.

We have already reported one of the events in this region, abnormal refraction or unidirectional wave energy flow. ${ }^{8}$ Our previous experiment showed enhancement of transmittance signal through a two-dimensional microplasma array without any amplification mechanism. This signal enhancement continues for more than $3 \mathrm{GHz}$ above the $30 \mathrm{GHz}$ region, and the wave measurement showed that divergence of wave energy flow was suppressed. Several evaluation results on electron density ${ }^{8,22,24}$ revealed that electron density was around $10^{13} \mathrm{~cm}^{-3}$, leading to the fact that $\omega_{\mathrm{pe}} / 2 \pi \sim 30 \mathrm{GHz}$. That is, the unusual signal enhancement was observed just above the electron plasma frequency.

We performed a numerical calculation using the direct solution of the wave equation using the finite difference method, ${ }^{8}$ in the similar way to what has been described in Sec. II B. Here we calculated the dispersion relation of propagating waves along the first band on a two-dimensional wave number plane. The derived equifrequency contours in general were circular shaped and showed isotropic wave propagation where the group velocity vector was in the direction of the phase velocity vector. However, in the vicinity of the electron plasma frequency, the equifrequency contours were not circular but square shaped, and anisotropy in wave propagation took place; the phase velocity in the $\Gamma-M$ direction becomes smaller than that in the $\Gamma-X$ direction, and almost all the group velocity vectors, estimated from gradient of the equifrequency contours $\operatorname{grad}_{k}\left[\omega\left(k_{x}, k_{y}\right)\right]$, points in the $\Gamma-X$ direction. When the waves are launched from a microwave horn antenna, the wave energy is likely to diverge somewhat in free space, but such divergence will be suppressed in the propagation in the two-dimensional microplasma array near the electron plasma frequency.

\section{Flatbands below electron plasma frequency}

In the frequency range less than the electron plasma frequency, flatbands are predicted in Figs. 1, 2, and 4 when the TE mode propagation is assumed. Since our experimental setup for wave detection was limited in the range more than $33 \mathrm{GHz}$ which is almost the electron plasma frequency in the previous condition, we increased the applied voltage for plasma production to elevate the electron plasma frequency and to set the flatbands region to be more than $33 \mathrm{GHz}$.

Figure 8 shows a transmittance feature in the TE mode in the lower frequency region than the first band gap, which was observed using an electrode system reported previously. ${ }^{7,8}$ There was transmittance variation intermittently with large amplitude from 0.1 to 3.0 at maximum within a very narrow frequency region $(0.1-1.0 \mathrm{GHz})$. This is attributed to the abrupt changes of the refractive index of the microplasma array and to the energy flow direction. Such a phenomenon is only possible in flatband regions at $\omega$ $<\omega_{\text {pe }}$ from the above theoretical predictions. Simple estimation of $n_{\mathrm{e}}$ by discharge currents with electron mobility in He from the literature ${ }^{25}$ yielded that $n_{\mathrm{e}}=(2-4) \times 10^{13} \mathrm{~cm}^{-3}$, which roughly agreed with the condition of $\omega=\omega_{\mathrm{pe}}$ at 40-45 GHz. Figure 8(b) clearly exhibits such a tendency. To 


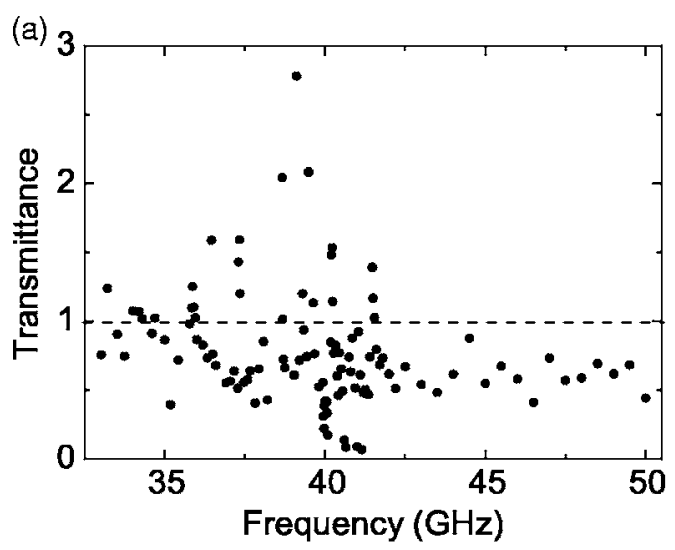

(b)

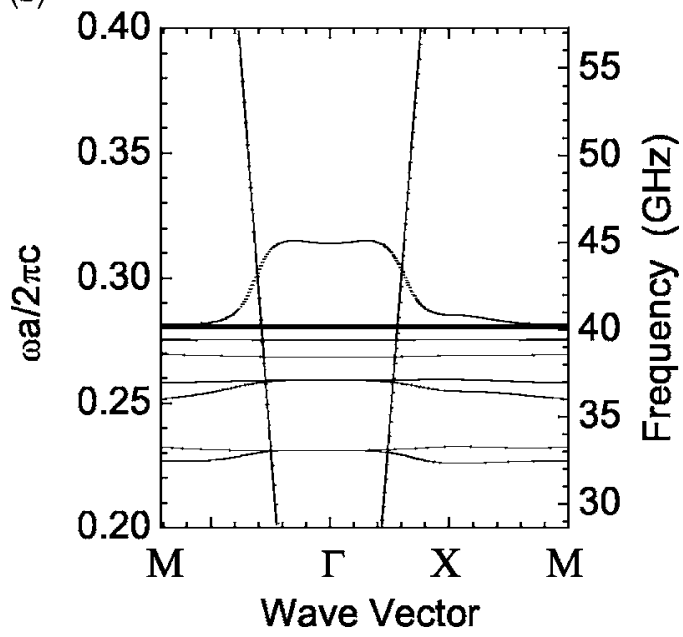

FIG. 8. (a) Transmittance of TE mode signals in the $\Gamma-X$ direction as a function of millimeter frequency. Electrode configuration was described in Refs. 7 and 8. (b) Enlarged band diagram in the case of lattice constant $a$ of $2.1 \mathrm{~mm}$ with 0.84 -mm-diameter plasma column of $n_{\mathrm{e}}=4 \times 10^{13} \mathrm{~cm}^{-3}$ and $\nu_{\mathrm{m}}=1.2 \omega_{\mathrm{pe}}$.

calculate this band structure, we set $n_{\mathrm{e}}=4 \times 10^{13} \mathrm{~cm}^{-3}$ and $\nu_{\mathrm{m}}=1.2 \omega_{\mathrm{pe}}$ to simulate atmospheric He discharges using the modified plane-wave expansion method described in Sec. II A. There are consistent features between this experimental result and the theoretical calculation; the observed swing of transmittance around $40 \mathrm{GHz}$ seems to correspond to the fine structures of the multiflatbands predicted in the same frequency region, and a monotonous tendency apart from this region is also common. However, more rigorous explanation is necessary to clarify their identification, such as the detailed equifrequency contours of each flatband in a twodimensional wave number plane. ${ }^{8}$

From the comparisons with Figs. 4(a) and 4(b), the fact that flatbands were localized at 37-42 GHz in Fig. 8(a) leads to evaluations that the $n_{\mathrm{e}}$ gradient on the edge was steep. There is another factor of this localization: the flatbands resulting from the edge low- $n_{\mathrm{e}}$ plasma regions might vanish since the $\nu_{\mathrm{m}}$ to $\omega_{\mathrm{pe}}$ ratio increased on the edges of the plasma columns due to lower $n_{\mathrm{e}}$, which has been suggested in Fig. 3 where the flatbands vanish at $\nu_{\mathrm{m}}>2 \omega_{\mathrm{pe}}$

\section{CONCLUDING REMARKS}

We demonstrated two theoretical approaches to uncover photonic bands in plasma photonic crystals. The plane-wave expansion method including frequency dependence revealed band diagrams with the effects of electron collision frequency. In collisionless plasma arrays, band gaps are formed as a function of lattice constant and filling fraction, similar to dielectric photonic crystals. Furthermore, multiflatbands emerge at lower frequencies than plasma frequency. Band gaps are affected less by electron elastic collisions, but multiflatbands disappear in a highly collisional regime. The direct solution of a wave equation using complex fields enabled us to obtain band diagrams of complicated unit structure, and the effects of electron density gradient were discussed. Calculated band diagrams are very similar to ones by the modified plane-wave expansion method, and we established two different methods to derive plasma photonic bands. Using this method, it was found that twodimensionally periodical plasma columns with electron density gradient enlarge the frequency range of multiflatbands and that calculated electric field profiles explain the physical property of each band. These theoretically derived dispersion properties agreed with observations in the experiments and will lead to further functional design of plasma photonic crystals.

\section{ACKNOWLEDGMENTS}

This work was supported by a Grant-in-Aid for Scientific Research from the Japanese Ministry of Education, Culture, Sports, Science and Technology and by the project to develop "innovative seeds" from the Japan Science and Technology Agency.

\section{APPENDIX: LINEARIZATION TECHNIQUE FOR A GENERALIZED EIGENVALUE PROBLEM FOR A COMPLEX MATRIX OF WAVE EXPANSION ${ }^{9}$}

From Maxwell equations with fields varying harmonically in time $t$ in the form of $\exp (j \omega t)$, we can expand the $z$ component of the magnetic field in the TE ( $H$ polarization) mode, where the $(x, y)$ plane exhibits the spatial periodicity of the dielectric coefficient as

$$
\boldsymbol{H}_{z}\left(\boldsymbol{x}_{\|} \mid \omega\right)=\sum_{G_{\|}} A\left(\boldsymbol{k}_{\|} \mid \boldsymbol{G}_{\|}\right) e^{j\left(\boldsymbol{k}_{\|}+\boldsymbol{G}_{\|}\right) \cdot \boldsymbol{x}_{\|}} .
$$

When we substitute Eq. (A1) into the wave equation about $H_{z}$, coefficients $\left\{A\left(\boldsymbol{k}_{\|} \mid \boldsymbol{G}_{\|}\right)\right\}$fulfill the following equation as

$$
\begin{aligned}
& \sum_{\boldsymbol{G}_{\|}{ }^{\prime}}\left(\boldsymbol{k}_{\|}+\boldsymbol{G}_{\|}\right) \cdot\left(\boldsymbol{k}_{\|}+\boldsymbol{G}_{\|}{ }^{\prime}\right) \hat{\kappa}\left(\boldsymbol{G}_{\|}-\boldsymbol{G}_{\|}{ }^{\prime}\right) A\left(\boldsymbol{k}_{\|} \mid \boldsymbol{G}_{\|}\right) \\
& \quad=\frac{\omega^{2}}{c^{2}} A\left(\boldsymbol{k}_{\|} \mid \boldsymbol{G}_{\|}\right),
\end{aligned}
$$

where $\left\{\hat{\kappa}\left(\boldsymbol{G}_{\|}\right)\right\}$are the Fourier coefficients of $1 / \varepsilon\left(\boldsymbol{x}_{\|} \mid \omega\right)$. Using Eqs. (2) and (3) and $\mu=\omega / c$, Eq. (A2) yields

$$
\mu^{4} \stackrel{\leftrightarrow}{\boldsymbol{I}}-\mu^{3} \stackrel{\leftrightarrow}{\boldsymbol{P}}-\mu^{2} \stackrel{\leftrightarrow}{\boldsymbol{Q}}-\mu \overleftrightarrow{\boldsymbol{R}}-\overleftrightarrow{\boldsymbol{S}}=0,
$$

where

$$
\stackrel{\leftrightarrow}{\boldsymbol{P}}\left(\boldsymbol{G}_{\|} \mid \boldsymbol{G}_{\|}{ }^{\prime}\right)=j \frac{\nu_{\mathrm{m}}}{c} \delta_{\boldsymbol{G}_{\|}, \boldsymbol{G}_{\|}{ }^{\prime}},
$$




$$
\begin{aligned}
\overleftrightarrow{\boldsymbol{Q}}\left(\boldsymbol{G}_{\|} \mid \boldsymbol{G}_{\|}{ }^{\prime}\right)= & \left\{\frac{\omega_{\mathrm{pe}}{ }^{2}}{c^{2}}+\left[\frac{1}{\varepsilon_{\mathrm{d}}}(1-f)+f\right] \cdot\left(\boldsymbol{k}_{\|}+\boldsymbol{G}_{\|}\right)^{2}\right\} \delta_{\boldsymbol{G}_{\|}, \boldsymbol{G}_{\|}{ }^{\prime}} \\
& +f\left(\frac{1}{\varepsilon_{\mathrm{d}}}-1\right)\left(\boldsymbol{k}_{\|}+\boldsymbol{G}_{\|}\right) \cdot\left(\boldsymbol{k}_{\|}\right. \\
& \left.+\boldsymbol{G}_{\|}{ }^{\prime}\right) \frac{2 J_{1}\left(\left|\boldsymbol{G}_{\|}-\boldsymbol{G}_{\|}{ }^{\prime}\right| R\right)}{\left|\boldsymbol{G}_{\|}-\boldsymbol{G}_{\|}{ }^{\prime}\right| R}, \\
\overleftrightarrow{\boldsymbol{R}}\left(\boldsymbol{G}_{\|} \mid \boldsymbol{G}_{\|}{ }^{\prime}\right)= & -j \frac{\nu_{\mathrm{m}}}{c}\left[\frac{1}{\varepsilon_{\mathrm{d}}}(1-f)+f\right]\left(\boldsymbol{k}_{\|}+\boldsymbol{G}_{\|}\right)^{2} \delta_{\boldsymbol{G}_{\|}, \boldsymbol{G}_{\|}{ }^{\prime}} \\
& -j \frac{\nu_{\mathrm{m}}}{c} f\left(\frac{1}{\varepsilon_{\mathrm{d}}}-1\right)\left(\boldsymbol{k}_{\|}+\boldsymbol{G}_{\|}\right) \cdot\left(\boldsymbol{k}_{\|}\right. \\
& \left.+\boldsymbol{G}_{\|}{ }^{\prime}\right) \frac{2 J_{1}\left(\left|\boldsymbol{G}_{\|}-\boldsymbol{G}_{\|}{ }^{\prime}\right| R\right)}{\left|\boldsymbol{G}_{\|}-\boldsymbol{G}_{\|}{ }^{\prime}\right| R}, \\
\overleftrightarrow{\boldsymbol{S}}\left(\boldsymbol{G}_{\|} \mid \boldsymbol{G}_{\|}{ }^{\prime}\right)= & -\frac{\omega_{\mathrm{pe}}{ }^{2}}{c^{2}} \frac{1}{\varepsilon_{\mathrm{d}}}(1-f)\left(\boldsymbol{k}_{\|}+\boldsymbol{G}_{\|}\right)^{2} \delta_{\boldsymbol{G}_{\|}, \boldsymbol{G}_{\|}{ }^{\prime}} \\
& +\frac{\omega_{\mathrm{pe}}{ }^{2}}{c^{2}} \frac{1}{\varepsilon_{\mathrm{d}}} f\left(\frac{1}{\varepsilon_{\mathrm{d}}}-1\right)\left(\boldsymbol{k}_{\|}+\boldsymbol{G}_{\|}\right) \cdot\left(\boldsymbol{k}_{\|}\right. \\
& \left.+\boldsymbol{G}_{\|}{ }^{\prime}\right) \frac{2 J_{1}\left(\left|\boldsymbol{G}_{\|}-\boldsymbol{G}_{\|}{ }^{\prime}\right| R\right)}{\left|\boldsymbol{G}_{\|}-\boldsymbol{G}_{\|}{ }^{\prime}\right| R},
\end{aligned}
$$

where the elements of the $N \times N$ matrices are $\boldsymbol{P}, \boldsymbol{Q}, \boldsymbol{R}$, and $\boldsymbol{S}$. This polynomial form is transformed into a linear problem in $4 N$ dimensions by $\boldsymbol{W}$ that fulfills

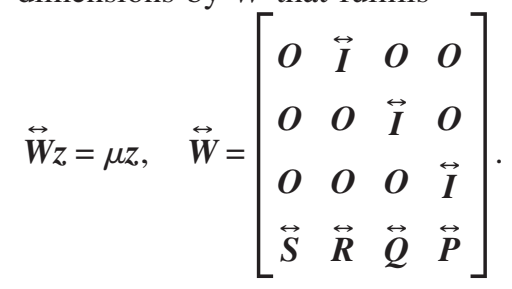

In the case of the TM ( $E$ polarization) mode, the $z$ component of electric field $E_{z}$ is

$$
\boldsymbol{E}_{z}\left(\boldsymbol{x}_{\|} \mid \omega\right)=\sum_{G_{\|}} B\left(\boldsymbol{k}_{\|} \mid \boldsymbol{G}_{\|}\right) e^{j\left(\boldsymbol{k}_{\|}+\boldsymbol{G}_{\|}\right) \cdot \boldsymbol{x}_{\|}}
$$

Components $\left\{B\left(\boldsymbol{k}_{\|} \mid \boldsymbol{G}_{\|}\right)\right\}$fulfill the following equation as

$$
\begin{aligned}
\left(\boldsymbol{k}_{\|}+\boldsymbol{G}_{\|}\right)^{2} B\left(\boldsymbol{k}_{\|} \mid \boldsymbol{G}_{\|}\right)= & \frac{\omega^{2}}{c^{2}} \hat{\varepsilon}(\mathbf{0}) B\left(\boldsymbol{k}_{\|} \mid \boldsymbol{G}_{\|}\right)+\frac{\omega^{2}}{c^{2}} \sum_{\boldsymbol{G}_{\|}{ }^{\prime}} \hat{\varepsilon}\left(\boldsymbol{G}_{\|}\right. \\
& \left.-\boldsymbol{G}_{\|}{ }^{\prime}\right) B\left(\boldsymbol{k}_{\|} \boldsymbol{G}_{\|}{ }^{\prime}\right),
\end{aligned}
$$

where $\Sigma_{\mathbf{G}_{\|}{ }^{\prime}}$ denotes summation except for the case with $\mathbf{G}_{\|}$ $=\mathbf{G}_{\|}{ }^{\prime}$. Using Eqs. (2) and (3), Eq. (A7) yields to

$$
\mu^{3} \overleftrightarrow{\boldsymbol{J}}-\mu^{2} \overleftrightarrow{\boldsymbol{K}}-\mu \overleftrightarrow{\boldsymbol{L}}-\overleftrightarrow{\boldsymbol{M}}=0
$$

where

$$
\begin{gathered}
\overleftrightarrow{\boldsymbol{J}}\left(\boldsymbol{G}_{\|} \mid \boldsymbol{G}_{\|}{ }^{\prime}\right)=\left[f+(1-f) \varepsilon_{\mathrm{d}}\right] \delta_{\boldsymbol{G}_{\|}, \boldsymbol{G}_{\|}{ }^{\prime}}+f(1 \\
\left.-\varepsilon_{\mathrm{d}}\right) \frac{2 J_{1}\left(\left|\boldsymbol{G}_{\|}-\boldsymbol{G}_{\|}{ }^{\prime}\right| R\right)}{\left|\boldsymbol{G}_{\|}-\boldsymbol{G}_{\|}{ }^{\prime}\right| R}
\end{gathered}
$$

$$
\begin{aligned}
\overleftrightarrow{\boldsymbol{K}}\left(\boldsymbol{G}_{\|} \mid \boldsymbol{G}_{\|}{ }^{\prime}\right)= & j \frac{\nu_{\mathrm{m}}}{c}\left\{\left[f+(1-f) \varepsilon_{\mathrm{d}}\right] \delta_{\boldsymbol{G}_{\|}, \boldsymbol{G}_{\|}{ }^{\prime}+f(1}\right. \\
& \left.\left.-\varepsilon_{\mathrm{d}}\right) \frac{2 J_{1}\left(\left|\boldsymbol{G}_{\|}-\boldsymbol{G}_{\|}{ }^{\prime}\right| R\right)}{\left|\boldsymbol{G}_{\|}-\boldsymbol{G}_{\|}{ }^{\prime}\right| R}\right\}, \\
\overleftrightarrow{\boldsymbol{L}}\left(\boldsymbol{G}_{\|} \mid \boldsymbol{G}_{\|}{ }^{\prime}\right)= & {\left[\left(\boldsymbol{k}_{\|}+\boldsymbol{G}_{\|}\right)^{2}+f \frac{\omega_{\mathrm{pe}}{ }^{2}}{c^{2}}\right] \delta_{\boldsymbol{G}_{\|}, \boldsymbol{G}_{\|}{ }^{\prime}} } \\
& +f \frac{\omega_{\mathrm{pe}}{ }^{2}}{c^{2}} \frac{2 J_{1}\left(\left|\boldsymbol{G}_{\|}-\boldsymbol{G}_{\|}{ }^{\prime}\right| R\right)}{\left|\boldsymbol{G}_{\|}-\boldsymbol{G}_{\|}{ }^{\prime}\right| R}, \\
\overleftrightarrow{\boldsymbol{M}}\left(\boldsymbol{G}_{\|} \mid \boldsymbol{G}_{\|}{ }^{\prime}\right)= & -j \frac{\nu_{\mathrm{m}}}{c}\left(\boldsymbol{k}_{\|}+\boldsymbol{G}_{\|}\right)^{2} \delta_{\boldsymbol{G}_{\|}, \boldsymbol{G}_{\|}{ }^{\prime}},
\end{aligned}
$$

where the elements of $N \times N$ matrices are $\boldsymbol{J}, \boldsymbol{K}, \boldsymbol{L}$, and $\boldsymbol{M}$. This polynomial form is transformed into a linear problem in $3 N$ dimensions by $\boldsymbol{U}$ and $\boldsymbol{V}$ that fulfills

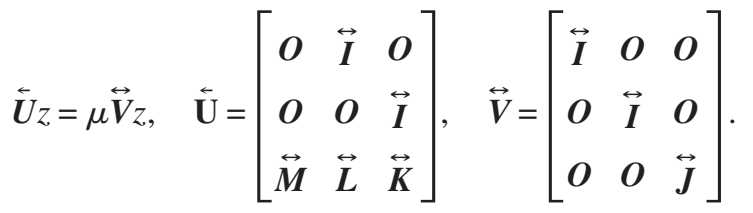

${ }^{1}$ E. Yablonovitch, Science 289, 557 (2000).

${ }^{2}$ Roadmap on Photonic Crystals, edited by S. Noda and T. Baba (Kluwer Academic, Boston, 2003).

${ }^{3}$ J. Faith, S. P. Kuo, and J. Huang, Phys. Rev. E 55, 1843 (1997).

${ }^{4}$ D. K. Kalluri, Electromagnetics of Complex Media (CRC, Boca Raton, 1998).

${ }^{5}$ H. Hojo and A. Mase, J. Plasma Fusion Res. 80, 89 (2004).

${ }^{6}$ H. Hojo, N. Uchida, and A. Mase, Plasma Fusion Res. 1, 21 (2006).

${ }^{7}$ O. Sakai, T. Sakaguchi, and K. Tachibana, Appl. Phys. Lett. 87, 241505 (2005).

${ }^{8}$ O. Sakai, T. Sakaguchi, Y. Ito, and K. Tachibana, Plasma Phys. Controlled Fusion 47, B617 (2005).

${ }^{9}$ V. Kuzmiak and A. A. Maradudin, Phys. Rev. B 55, 7427 (1997).

${ }^{10}$ K. Sakoda, N. Kawai, T. Ito, A. Chutinan, S. Noda, T. Mitsuyu, and K. Hirano, Phys. Rev. B 64, 045116 (2001).

${ }^{11}$ E. Moreno, D. Erni, and C. Hafner, Phys. Rev. B 65, 155120 (2002).

${ }^{12}$ O. Toader and S. John, Phys. Rev. E 70, 046605 (2004).

${ }^{13}$ R. L. Chern, C. C. Chang, and C. Chung Chang, Phys. Rev. E 73, 036605 (2006).

${ }^{14}$ J. B. Pendry, A. J. Holden, W. J. Stewart, and I. Young, Phys. Rev. Lett. 76, 4773 (1996).

${ }^{15}$ Y. Noguchi, A. Matsuoka, K. Uchino, and K. Muraoka, J. Appl. Phys. 91, 613 (2002).

${ }^{16}$ K. M. Ho, C. T. Chan, and C. M. Soukoulis, Phys. Rev. Lett. 65, 3152 (1990).

${ }^{17}$ M. Phihal, A. Shambrook, A. A. Maradudin, and P. Sheng, Opt. Commun. 80, 199 (1991)

${ }^{18}$ T. Ito and K. Sakoda, Phys. Rev. B 64, 045117 (2001).

${ }^{19}$ O. Sakai and K. Tachibana, IEEE Trans. Plasma Sci. 34, 80 (2006).

${ }^{20}$ K. S. Kunz and R. J. Luebbers, The Finite Difference Time Domain Method for Electromagnetics (CRC, Boca Raton, 1993); J. H. Lee and D. K. Kalluri, IEEE Trans. Antennas Propag. 47, 1146 (1999).

${ }^{21}$ E. E. Kunhaldt, IEEE Trans. Plasma Sci. 28, 189 (2000).

${ }^{22}$ T. Sakaguchi, O. Sakai, and K. Tachibana, J. Appl. Phys. 101, 073305 (2007), this issue.

${ }^{23}$ W. M. Robertson, G. Arjavalingam, R. D. Maede, K. D. Brommer, A. M. Rappe, and J. D. Joannopoulos, Phys. Rev. Lett. 30, 2023 (1992).

${ }^{24}$ O. Sakai, Y. Kishimoto, and K. Tachibana, J. Phys. D 38, 431 (2005).

${ }^{25}$ S. C. Brown, Basic Data of Plasma Physics (MIT Press, Boston, 1959). 